\section{Respuesta a Carta al Editor}

\section{Sr. Editor}

En relación a la carta titulada: Evidencias de la capacidad predictiva del Child Behavior Checklist 1.5-5 (CBCL/1.5-5) queremos hacer las siguientes precisiones.

El proceso de validez de un instrumento se construye a lo largo del tiempo, en distintas muestras, poblaciones y por grupos de investigadores independientes al grupo original que validó el instrumento. Por lo mismo suele ser un proceso largo y costoso ${ }^{1}$.

En ese sentido el CBCL 1.5-5 es uno de los instrumentos para medir la psicopatología más validados en distintos países ${ }^{2-5}$. Además, la uniformidad de la metodología usada por los investigadores para evaluar las propiedades psicométricas del CBCL ha hecho que la comparación transcultural sea posible ${ }^{6}$.

En la mayoría de los estudios de validez de este instrumento la curva ROC se realiza para demostrar la validez de criterio (referidos clínicamente vs no referidos clinicamente). En nuestro estudio el puntaje total crudo de 24 fue el mejor punto de corte a partir de la curva ROC y el valor con mejor sensibilidad y especificidad. Sin embargo, nuestro resultado con un ABC (área bajo la curva) en el límite de lo aceptable de 0,77 nos obliga a considerar varias posibilidades: 1 ) la referencia clínica puede no reflejar lo mismo en países desarrollados que en países con una limitada infraestructura de salud mental como México; 2) el estigma asociado a los trastornos psiquiátricos pudiera influir en la decisión de referir a los niños para una valoración de salud mental; 3) el personal que hace la referencia clínica pudiera tener conocimientos limitados sobre cuales problemas de salud mental de los niños entre 1 y 5 años de edad ameritan intervención y tratamiento. Nuestro estudio de validez inicial de la versión mexicana del CBCL/1.55 deja varios análisis pendientes que deben completar- se en estudios futuros como serían la realización de un análisis factorial confirmatorio, la confiabilidad interevaluador entre distintos informantes (padre-madre), la validez de constructo de cada uno de los síndromes que explora el instrumento, la validez concurrente con otros instrumentos de psicopatología y la sensibilidad al cambio. A pesar de estas limitaciones consideramos que el CBCL/1.5-5 es un instrumento útil y necesario que puede utilizarse en México para propósitos clínicos y de investigación.

\section{Referencias}

1. Kimberlin CL, Winterstein AG. Validity and reliability of measurement instruments used in research. Am J Health Syst Pharm. 2008;65(23):2276-84.

2. Lecannelier F, Pérez Ewert JC, Groissman S, Gallardo D, Bardet AM, Bascuñan A, et al. Validación del Inventario de Conductas Infantiles para niños de entre 11/2-5 años (CBCL 11/2-5) en la Ciudad de Santiago. Univ Psychol [Internet]. 2014;13(2). Available from: http://revistas.javeriana.edu.co/ index.php/revPsycho/article/view/1610 [citado el 1 de marzo de 2017].

3. Pandolfi V, Magyar CI, Dill CA. Confirmatory factor analysis of the child behavior checklist 1.5-5 in a sample of children with autism spectrum disorders. J Autism Dev Disord. 2009;39(7):986-95.

4. Liu J, Cheng H, Leung PWL. The Application of the Preschool Child Behavior Checklist and the Caregiver-Teacher Report Form to Mainland Chinese Children: Syndrome Structure, Gender Differences, Country Effects, and Inter-Informant Agreement. J Abnorm Child Psychol. 2011;39(2):251-64.

5. Muratori F, Narzisi A, Tancredi R, Cosenza A, Calugi S, Saviozzi I, et al. The CBCL 1.5-5 and the identification of preschoolers with autism in Italy. Epidemiol Psychiatr Sci. 2011;20(4):329-38.

6. Ivanova MY, Achenbach TM, Rescorla LA, Harder VS, Ang RP, Bilenberg N, et al. Preschool Psychopathology Reported by Parents in 23 Societies: Testing the Seven-Syndrome Model of the Child Behavior Checklist for Ages 1.5-5. J Am Acad Child Adolesc Psychiatry. 2010;49(12):1215-24.

Dra. Lilia Albores-Gallo 